https://helda.helsinki.fi

\title{
Optical position clamping with predictive control
}

\section{Ojala, Heikki}

Applied Physics Letters

2009-11-04

Appl. Phys. Lett. 95, 181104 (2009)

http://link.aip.org/link/?apl/95/181104

Downloaded from Helda, University of Helsinki institutional repository.

This is an electronic reprint of the original article.

This reprint may differ from the original in pagination and typographic detail.

Please cite the original version. 


\title{
Optical position clamping with predictive control
}

\author{
Heikki Ojala, Anders Korsbäck ${ }^{*}$, Anders E. Wallin, and Edward Hæggström
}

Department of Physics, University of Helsinki, POBox 64, FIN-00014, Helsinki, FINLAND

\begin{abstract}
:
We increase the effective stiffness of optical tweezers by position clamping a polystyrene bead with a predictive feedback control algorithm. This algorithm mitigates the effect of feedback loop delay. Hence, higher gain than with proportional control can be employed, which results in higher effective trap stiffness, without trap instability. In experiments (initial trap stiffness $0.056 \mathrm{pN} / \mathrm{nm}$ with a $1.78 \mu \mathrm{m}$ diameter polystyrene bead) predictive control increased the effective trap stiffness by 55\% relative to proportional control. We also derive theoretical expressions for the power spectra of the bead position controlled by our algorithm.
\end{abstract}

Key-words: optical tweezers, feedback control, radiation pressure, acousto-optic deflectors

PACS: 42.50.Wk, 87.80.Cc, 02.30.Yy

*e-mail: anders.korsback@helsinki.fi

Copyright (2009) American Institute of Physics. This article may be downloaded for personal use only. Any other use requires prior permission of the author and the American Institute of Physics

The following article appeared in (Appl. Phys. Lett. 95, 181104 (2009)) and may be found at (http://link.aip.org/link/?apl/95/181104) 
Optical tweezers are regularly used in single-molecule biophysical experiments, where the sample is attached to an optically trapped silica or polystyrene bead used as a handle ${ }^{1}$. Feedback control can increase the stiffness of optical tweezers in position clamp mode ${ }^{2-6}$. Wulff et al. used an adaptive filter and fast steering mirrors for control ${ }^{4}$. This adaptive control reduced the rmsdisplacement by $90 \%$ compared to no feedback, albeit only in the low frequency region (below $90 \mathrm{~Hz}$ ). Simulations ${ }^{5}$ showed similar improvements as our previous measurements ${ }^{2}$. Ranaweera et al. estimated a 29-fold increase in stiffness with simple proportional control and a 65-fold increase with a nonlinear controller. However, these simulations did not take loop delay, the delay between measurement and control, into account. Simmons et al. measured a 400-fold increase in stiffness with Proportional- Integral- Derivative-control (PID) and 5 kHz bandwidth ${ }^{6}$. The achievable improvement in effective stiffness by means of proportional feedback is limited by the loop delay ${ }^{2}$. In this letter we present a predictive control algorithm that mitigates the destabilizing effect of the loop delay, introduce a theory describing the effects of the algorithm, and experimentally measure the increase in effective trap stiffness. This algorithm is specifically designed with the dynamics of the controlled system in mind, and outperforms traditional PIDcontrol $^{2}$.

The power spectral density (PSD) of bead position characterizes optical trapping ${ }^{7}$. Simple proportional control with loop delay $\tau$ and feedback gain $G$ gives a PSD $^{2}$ :

$$
|\tilde{x}(\omega)|^{2}=\frac{4 k_{B} T \gamma}{|i \omega \gamma+k+k G \exp (-i \omega \tau)|^{2}},
$$

where $\gamma$ is the drag coefficient, $k$ is the trap stiffness, $x$ is the bead position, $T$ is the temperature, and $k_{B}$ is the Boltzmann constant. From this expression we can calculate the effective trap stiffness, which we define by the equipartition theorem:

$$
k_{\text {eff }}=\frac{k_{B} T}{\left\langle x^{2}>\right.}=\frac{k_{B} T}{\int_{0}^{\infty}|\tilde{x}(\omega)|^{2} \mathrm{~d} \omega} .
$$

The PSD described by Eq. (1) reduces to a Lorentzian when $G=0$. Increasing $G$ lowers the level of the low-frequency part of the spectrum, but causes a resonance peak to appear around $10 \mathrm{kHz}$ (when $\tau \approx 15 \mu \mathrm{s}$ ). Eventually, the growth of the resonance peak dominates the PSD, and the effective stiffness decreases. The resonance peak is a result of the loop delay. The bead moves during the delay and the trap position determined one loop delay time ago may be an inappropriate estimate of the current bead position.

The predictive control algorithm introduced here differs from proportional control in that it determines trap position not according to the current measured value of the bead position, but estimates the bead position one loop delay time into the future, so that for proportional control, ideally

$$
x_{\text {trap }}(t+\tau)=-G x(t+\tau),
$$

similarly to the practice of "leading the target". This is impossible to do exactly due to random thermal noise. However, assuming zero thermal noise, and since $x_{\text {trap }}$ is constant during one sampling period $\Delta$, we can solve the equation of motion and obtain 


$$
x(t+\Delta)=x(t)-\alpha\left[x(t)-x_{\text {trap }}(t)\right],
$$

where $\alpha=1-\exp (-k \Delta / \gamma)$. In our hardware $\Delta=5 \mu$ s and the loop delay time $\tau \approx 15 \mu \mathrm{s}$. We assume that three sampling periods will pass during the loop delay time. By applying Eq. (4) iteratively we get

$$
x(t+\tau)=x(t)-\alpha\left[\left(\alpha^{2}-3 \alpha+3\right) x(t)-x_{\text {trap }}(t+2 \Delta)-(1-\alpha) x_{\text {trap }}(t+\Delta)-\left(\alpha^{2}-2 \alpha+1\right) x_{\text {trap }}(t)\right] .
$$

With typical experimental parameters $\alpha$ is expected to be 0 to 0.05 , whereby higher powers of $\alpha$ vanish. By dropping $\alpha$-terms of second or higher order, and inserting into Eq. (3) we obtain our predictive control expression. This expression is an infinite impulse response filter with three previous output signals and the latest input signal:

$$
x_{\text {trap }}(t)=-G\left\{x(t-\tau)-\alpha\left[3 x(t-\tau)-x_{\text {trap }}(t-\Delta)-x_{\text {trap }}(t-2 \Delta)-x_{\text {trap }}(t-3 \Delta)\right]\right\} .
$$

Simulations showed that including higher-order terms of $\alpha$ in the expression did not noticeably affect the improvement of effective stiffness. Equation (6) can be Fourier-transformed to obtain a theoretical expression for the PSD of the position of a trapped bead:

$$
|\tilde{x}(\omega)|^{2}=\frac{4 k_{B} T \gamma}{\left|i \omega \gamma+k+\frac{k(1-3 \alpha) \exp (-i \omega \tau)}{\frac{1}{G}+\alpha(\exp (-i 3 \omega \Delta)+\exp (-i 2 \omega \Delta)+\exp (-i \omega \Delta))}\right|^{2}} .
$$

which reduces to Eq. (1) when $\alpha=0$.

Predictive control was experimentally tested using a setup similar to that $i^{2}$. Polystyrene beads $\left(1.78 \mu \mathrm{m}\right.$ diameter, Kisker) were suspended in water at room temperature $\left(22^{\circ} \mathrm{C}\right)$. This solution was injected into a custom made chamber (50 $\mu \mathrm{m}$ deep, $2 \mathrm{~mm}$ wide) on an inverted microscope (TE-2000-U, Nikon). The microscope objective (TIRF 100x, 1.49 NA, Nikon) forms the trap using a $1064 \mathrm{~nm} \mathrm{CW}$ laser beam (Compass 1064-4000M, Coherent). The trapping laser beam was steered with two perpendicular acousto-optical deflectors (AODs) (45035-3-6.5DEG1.06-XY, Neos Technologies) controlled with direct digital frequency synthesizers (DDS) (64010-200-2AMDFS, Neos Technologies). The DDS require a 30-bit control word which was formed with a Field Programmable Gate Array (FPGA) card (PCI-7833R, National Instruments) where the control algorithm runs. Position detection of the trapped beads was done with two detection lasers (785 nm, HL7851G, Hitachi and $830 \mathrm{~nm}$, DL5032-001, Thorlabs) using position sensitive detectors (S2-0171, SiTek) in the back-focal plane. The detector beams were aligned on top of each other to allow two independent position measurements of the trapped particle: an inloop signal was used for control and an out-of-loop signal was recorded for verification of results. All measurement data used in this letter is from the out-of-loop detector. By scanning a trapped bead with the AODs through the focal area of the detector lasers and by performing a linear fit, the 30-bit control word of the AOD was related to the position signal. Bead position data was then collected at $200 \mathrm{kS} / \mathrm{s}$ for $3 \mathrm{~s}$ with each combination of control parameter values $(\alpha, G)$. From these time-series the PSDs were calculated, and Eq. (7) was fit to the data. Before 
fitting, the PSDs were blocked using logarithmic frequency intervals and the fits were carried out at $100 \mathrm{~Hz}-50 \mathrm{kHz}$ on a logarithmically scaled y-axis.
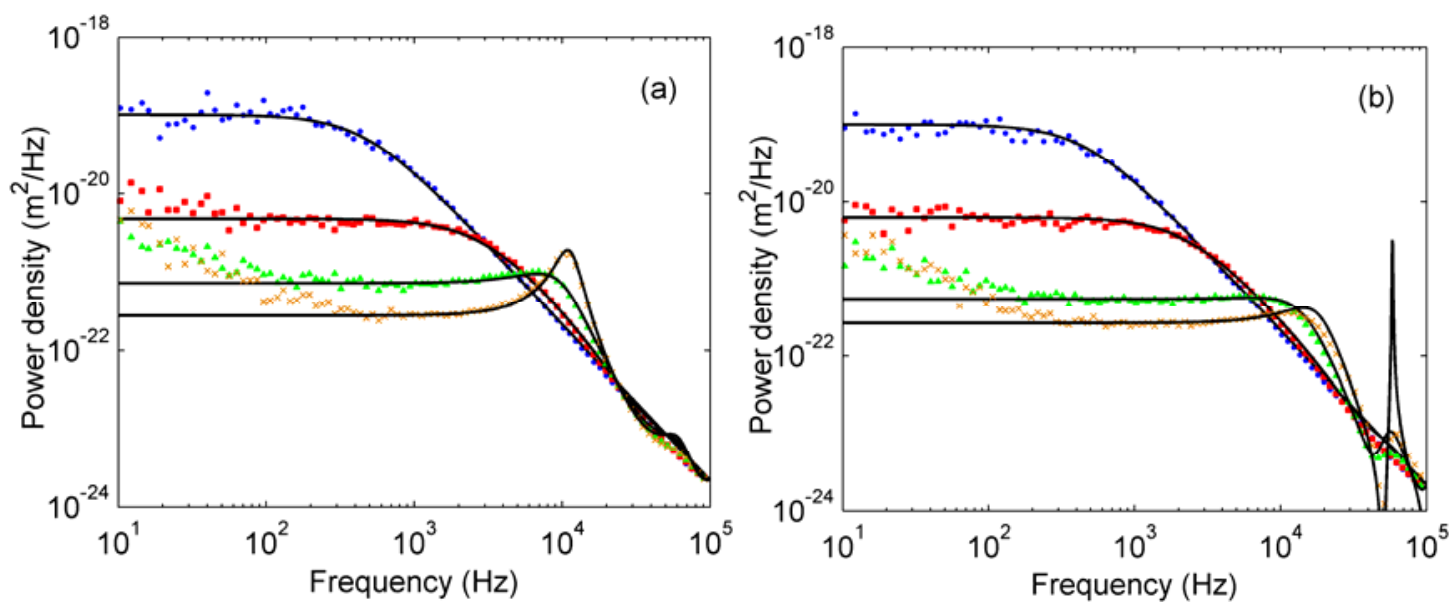

FIG. 1. (Color online) Bead position power spectra for (a) proportional ( $\alpha=0$ ) and (b) predictive ( $\alpha=0.013$ ) control. (a) spectra for gain values 0 (blue dots), 3.6 (red squares), 10.9 (green triangles), and 18.1 (brown crosses). (b) spectra for gain values 0 (blue dots), 3.6 (red squares), 27.2 (green triangles) and 79.8 (brown crosses). Black solid lines are theoretical predictions from Eq. (7) for different gain values and fixed other parameter values.

Figure 1(a) shows how increased gain introduces a resonance peak (around $10 \mathrm{kHz}$ ) to the PSD when using proportional control $(\alpha=0)$. In contrast, the predictive control $(\alpha=0.013)$ removes the resonance peak (Fig. 1(b)) and allows higher feedback gain. All bead position histograms remained Gaussian. Experimental and theoretical PSDs agree well for both proportional and predictive control. At the highest frequencies the measured power was damped due to the bandwidth limitations of the photodetectors ${ }^{8}$. At the low-frequency end, the spectrum shows 1/f-noise which is not found in the in-loop detector signal (data not shown).

Figure 2 shows how effective stiffness, calculated from Eq. (2), for proportional control reaches its maximum at $G=12.7$ and then decreases due to the resonance peak in the PSD. Since predictive control removes this resonance peak, effective stiffness keeps increasing up to $G=79.8$. Inserting Eq. (7) into Eq. (2) using previously determined parameter values and integrating to $50 \mathrm{kHz}$ we obtain a theoretical prediction for the effective trap stiffness. The $50 \mathrm{kHz}$ limit was chosen to make the theoretical prediction and measured stiffness comparable considering detector bandwidth. 


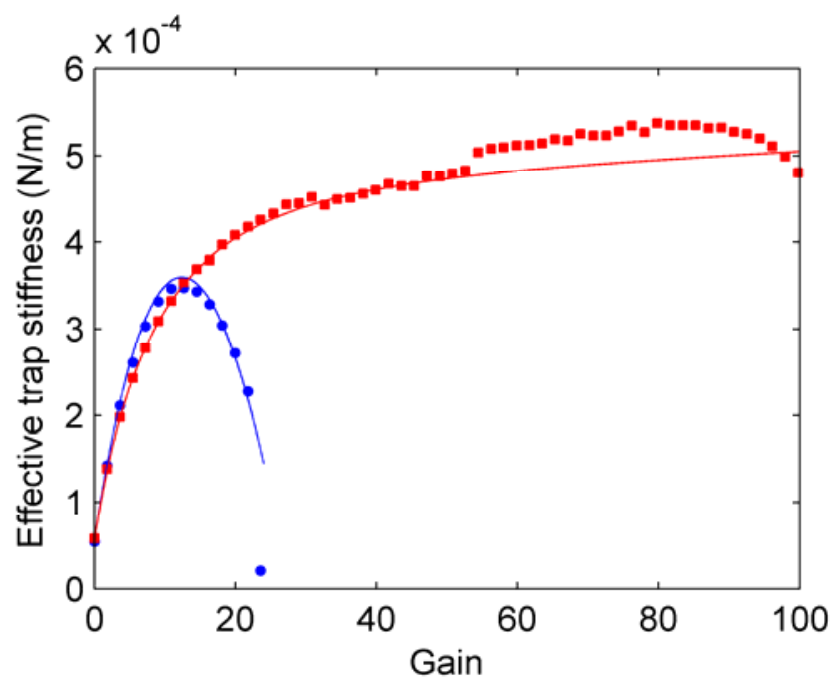

FIG. 2. (Color online) Effective stiffness vs. gain for proportional (blue dots, $\alpha=0$ ) and predictive (red squares, $\alpha=0.013$ ) control. Points are measured values computed from a recorded timeseries by means of Eq. (2), whereas solid lines are theoretical predictions calculated from Eq. (7) and Eq. (2) using the parameters determined by the PSD fits and integrating to $50 \mathrm{kHz}$ in Eq. (2).

The initial trap stiffness, without feedback control, was $0.056 \mathrm{pN} / \mathrm{nm}$. Proportional control increased the effective stiffness by a factor 6.2 to $0.35 \mathrm{pN} / \mathrm{nm}$ when $G=12.7$, whereas predictive control improved stiffness by a factor 9.6 to $0.54 \mathrm{pN} / \mathrm{nm}$ when $G=79.8$. Thus, predictive control offered an improvement in stiffness of 55\% compared to proportional control. As seen from Eq. (1) and Eq. (7), one way to improve the feedback control is to reduce the loop delay time. This time arises from the acoustic time-of-flight in the AOD and from the analog-todigital (AD) conversion in the FPGA. The time-of-flight in the AOD is difficult to improve but faster $\mathrm{AD}$-converters exist. However, the $\mathrm{AD}$-conversion is responsible only for the one third of the total loop delay time. A drawback of our predictive feedback control algorithm is that the optimal value of the $\alpha$-parameter depends on bead size, initial trap stiffness, and the viscosity.

In this letter we introduced a predictive feedback control algorithm for position clamping. With this control we were able to increase the effective stiffness by $55 \%$ compared to proportional control. Position clamping can be used to increase the trap stiffness in applications where increased laser light may cause sample heating ${ }^{9}$, photobleaching ${ }^{10}$ or photodamage to the samples $^{11}$. Knowing the system dynamics attained from the position clamp may also allow designing more precise active force clamps.

This work was supported by an Academy of Finland grant (\#128518), and HENAKOTO funds from the University of Helsinki. H.O. is supported by the Väisälä Fund (Finnish Academy of Science and Letters). 


\section{References}

1 T. T. Perkins, Laser \& Photonics Review 3, 203 (2009).

2 A. E. Wallin, H. Ojala, E. Hæggstrom, and R. Tuma, Applied Physics Letters 92, 224104 (2008).

$3 \quad$ K. D. Wulff, D. G. Cole, and R. L. Clark, Applied Optics 46, 4923 (2007).

$4 \quad$ K. D. Wulff, D. G. Cole, and R. L. Clark, Applied Optics 47, 3585 (2008).

5 A. Ranaweera and B. Bamieh, International Journal of Robust and Nonlinear Control 15, 747 (2005).

$6 \quad$ R. M. Simmons, J. T. Finer, S. Chu, and J. A. Spudich, Biophysical Journal 70, 1813 (1996).

$7 \quad$ K. Berg-Sørensen and H. Flyvbjerg, Review of Scientific Instruments 75, 594 (2004).

8 J. H. G. Huisstede, B. D. van Rooijen, K. O. van der Werf, M. L. Bennink, and V. Subramaniam, Optics Letters 31, 610 (2006).

9 E. J. G. Peterman, F. Gittes, and C. F. Schmidt, Biophysical Journal 84, 1308 (2003).

10 M. J. Lang, P. M. Fordyce, A. M. Engh, K. C. Neuman, and S. M. Block, Nature Methods 1, 133 (2004).

11 M. B. Rasmussen, L. B. Oddershede, and H. Siegumfeldt, Applied and Environmental Microbiology 74, 2441 (2008). 